\title{
Synovial remodelling and vascularization by Dickkopf-1 in osteoarthritis
}

D

ata from experimental models of osteoarthritis (OA) and human tissue samples reveal that Dickkopf-related protein 1 (also known as Dickkopf-1, DKK1) “impedes synovial homeostasis in OA joint compartments," according to Feng-Sheng Wang, an author of a new study published in Arthritis \& Rheumatism. "Loss of Dkk1 function reduces the secretion of proangiogenic and cartilage-proteolytic factors, stabilizes synovial integrity and eventually protects against articular cartilage damage," adds Wang.

DKK1 inhibits Wnt signalling and is implicated in cartilage homeostasis, skeletal remodelling and joint disorders. Loss of Dkk1 function in animal models of OA impedes pathogenesis, in a disease characterized by aberrant synovial vascularization and cartilage degradation. DKK1 has been implicated in neovascularization in cancer, but the trigger for angiogenesis in OA was unclear, and the role of DKK1 in this regard was unknown.

The investigators used synovial tissue harvested after total joint arthroplasty from 25 patients with end-stage $\mathrm{OA}$, as well as 21 control patients who had no radiographic OA. They also studied human chondrocytes and synovial fibroblasts in cell culture, and a rat model of surgicallyinduced $\mathrm{OA}$.

Immunostaining revealed greater vascularity and higher expression of DKK1 in OA samples than controls, and DKK1 expression was associated with expression of angiogenic factors and cartilage proteolytic enzymes. Treatment with the proinflammatory cytokine IL- $1 \beta$ increased DKK1 expression in synovial fibroblasts, and vice versa, and IL-1 receptor antagonism reduced expression of DKK1. Antibodies to DKK1 reduced IL- $1 \beta$-mediated upregulation of the expression of angiogenic and cartilagedegrading factors in cell culture, and reduced tube formation by human umbilical cord vascular endothelial cells.
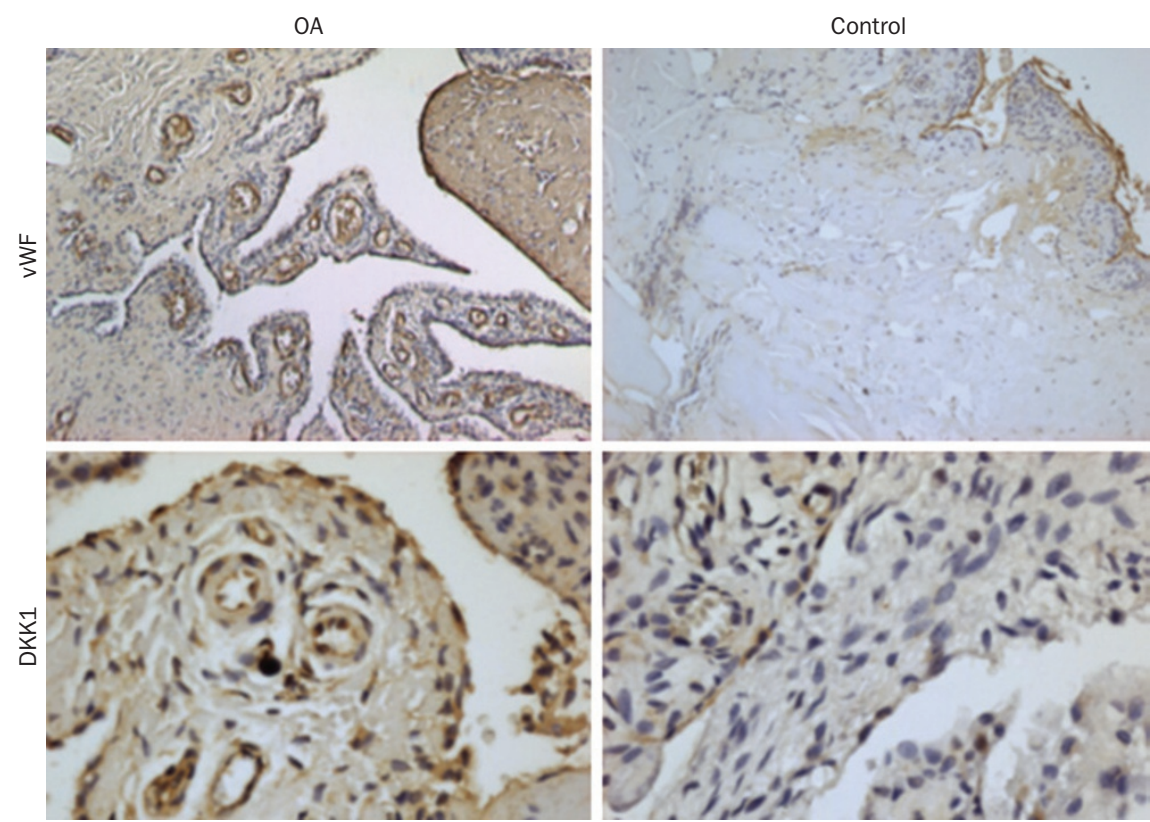

Synovia from patients with OA display hypervascularity and inflammation, with strong immunostaining of DKK1 compared with control synovia from patients with knee injury without OA. Blood vessels in these panels are shown by vWF immunostaining. Abbreviations: DKK1, dickkopf-related protein 1; OA, osteoarthritis; vWF, von Willebrand factor. Image courtesy of F.-W. Wang.

In a rat surgical model of $\mathrm{OA}$, a synthetic antisense oligonucleotide targeting the coding region of Dkk1 mRNA reduced the expression of Dkk1, angiogenic factors and proteinases in OA knees, and reduced OA-associated hypervascularization and cartilage pathology.

"This is really interesting work," opines David Walsh, an expert in angiogenesis and OA from the Arthritis Research UK Pain Centre, University of Nottingham, UK. "The paper by Weng et al. adds to a growing body of evidence that treatments known to target angiogenesis may reduce progression of structural damage in osteoarthritis." Walsh cautions, however, that Dkk1 is a pleiotropic mediator, and that it is "uncertain by which mechanisms Dkk1 knockdown improves joint pathology in the authors' surgical model of OA." Off-target effects might therefore be a concern. Rik Lories, who specializes in signalling between tissues in chronic arthritis at KU Leuven, Belgium, agrees. "Blocking DKK1 in OA patients may also have undesired effects on the bone, osteophytes and cartilage," he says. Furthermore, an apparent upstream role for IL-1 makes it "unclear whether targeting DKK1 would be beneficial in the case of ongoing inflammation in the human setting," Lories continues, adding "although the rat data are interesting."

Wang is optimistic. "We anticipate that Dkk1 will be a potent biochemical marker to diagnose the occurrence of knee OA. Moreover, reduction of Dkk1 action will be a feasible strategy to improve OA-induced joint damage or vessel disorders in the future," he concludes.

Emma Leah 